\section{Novel mutation in $A B C C 6$ gene in a Japanese pedigree with pseudoxanthoma elasticum and retinitis pigmentosa}

\begin{abstract}
Purpose To report a novel mutation of the $A B C C 6$ gene in a Japanese family that had a case of pseudoxanthoma elasticum (PXE) another with PXE and retinitis pigmentosa. Methods Ophthalmologic examinations were performed, and the $A B C C 6$ gene was analysed by direct genomic sequencing.

Results Fundus examinations of the 48-yearold proband disclosed angioid streaks and a peud'orange apparance of the retina of the both eyes, whereas both of his 25- and 20-yearold daughters had pigmentary degeneration and angioid streaks. In the sibilings, the mixed cone-rod ERG was almost nondetectable, whereas that of the proband was wellpreserved. Molecular genetic analysis revealed that the proband has a homozygous nonsense mutation at the $595 \mathrm{bp}$ in the $A B C C 6$, and the siblings were heterozygous for the same mutation. This mutation was not detected in Japanese subjects in the JSNP database (http:// snp.ims.u-tokyo.ac.jp/).

Conculsions Our results demonstrated an association between a novel mutation in the ABCC6 gene and PXE in a Japanese family. Eye (2005) 19, 215-217. doi:10.1038/sj.eye.6701449 Published online 21 May 2004
\end{abstract}

Keywords: ATP-binding cassette transporter; angioid streaks; molecular genetic analysis; pseudoxanthoma elasticum; retinitis pigmentosa

Pseudoxanthoma elasticum (PXE) is an autosomal inherited disease characterized by progressive dystrophic calcification of the
S Yoshida', M Honda', A Yoshida', S Nakao', Y Goto ${ }^{2}$, T Nakamura' ${ }^{1}$ K Fujisawa ${ }^{1}$ and T Ishibashi ${ }^{1}$ elastic structures in the skin, eyes, and cardiovascular system. ${ }^{1}$ The ocular abnormalities include peau d'orange or mottled hyperpigmentation of the retina, comet-like streaks, pinpoint white lesions of the choroid, and angioid streaks. PXE is caused by mutations in the ATP-binding cassette transporter, ABCC6. ${ }^{1,2}$ Although several cases of PXE, with different degrees of severity, manifestations, and mode of inheritance, have been reported in the Western world, the genetic aetiology is unknown in the eastern world. ${ }^{3}$

\section{Case reports}

The proband (I:1) and two daughters (II:2 and II:3), a two-generation Japanese family with no history of consanguinity, were examined ophthalmologically and genetically (Figure 1a). The visual acuity of the 48-year-old father was 20/100 OD and 20/250 OS, whereas that of his 25- and 20-year-old daughters was 20/20 OU. However, both daughters complained of nightblindness. Fundus examination of the proband disclosed subretinal proliferation due to angioid streaks (Figure 1b), and the peripheral retina had a mottled appearance (peu d'orange) in both eyes. In contrast, the siblings had bilateral pigmentary degeneration of retina compatible with retinitis pigmentosa (RP) with angioid streaks (Figure 1c and d). Family history indicated that there were no other members who had RP.

In parallel with the fundus appearance, Goldmann kinetic perimetry of the father revealed central scotomas, whereas the siblings demonstrated restricted visual fields. The scotopic rod and $30 \mathrm{~Hz}$ flicker ERGs were very
${ }^{1}$ Department of Ophthalmology, Kyushu University, Graduate School of Medicine, Fukuoka Japan

${ }^{2}$ Clinical Neurophysiology Kyushu University Graduate School of Medicine Fukuoka, Japan

Correspondence: S Yoshida Department of Ophthalmology, Kyushu University Graduate School of Medicine

Fukuoka 812-8582, Japan

Tel: + 81926425648

Fax: + 81926425663

E-mail: yosida@

med.kyushu-u.ac.jp

Received: 6 September 2003

Accepted: 6 January 2004 Published online: 21 May 2004 
reduced in the siblings, whereas those of the father were relatively well-preserved (Figure 2a).

Except for the younger sister, the father and older daughter had apparent cutaneous manifestations of PXE,

a
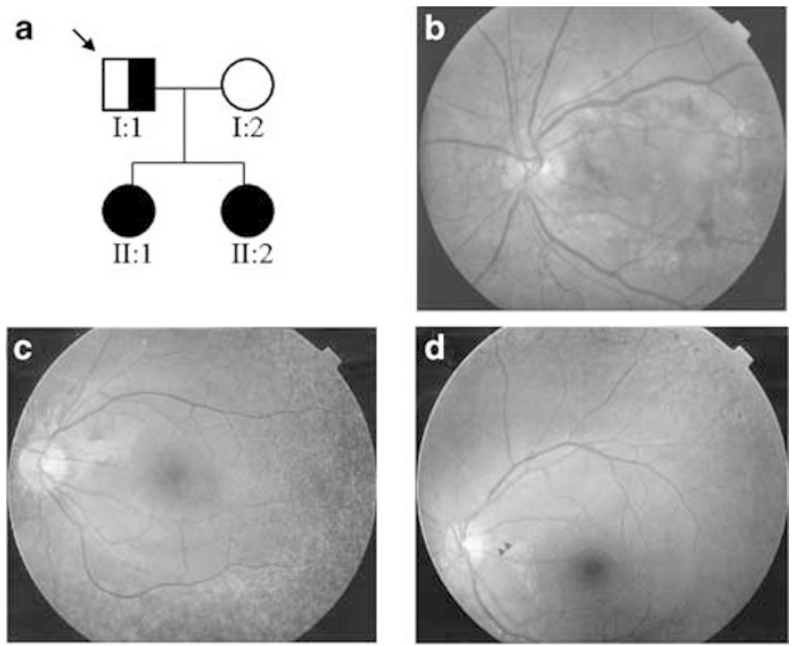

Figure 1 (a) Pedigree of the family. Solid black = affected members with pseudoxanthoma elasticum (right) and retinitis pigmentosa (left); white symbol = unaffected members. All members in the pedigree were examined by mutation screening, and the arrow indicates the proband. (b) Fundus photographs (left eye) of the proband showing remarkable angioid streaks radiating from the optic disc. Subretinal haemorrhage, choroidal neovascularization, and serous retinal detachment are observed in the macula. Fundus photograph of the left eye of (c) patient II:1 and (d) patient II:2, showing peau d'orange and/or pigmentary degenerative changes in the peripheral retina. Peripheral vessels are slightly attenuated. Angioid streaks (arrowhead) are also seen. The disease of patient II: 1 was more severe than II:2. but they had no cardiovascular involvement. Based on their clinical appearance, their illness was diagnosed as PXE.

After obtaining informed consent, molecular genetic analysis $^{4}$ of the $A B C C 6$ gene by direct sequencing of the 31 exons revealed that the proband had a homozygous and the two siblings had a heterozygous nonsense mutation ( $\mathrm{C} \rightarrow \mathrm{T}$ substitution) at $595 \mathrm{bp}$ in $A B C C 6$ (Figure $2 \mathrm{~b}$ ). This mutation produces a stop codon at 199, which is predicted to result in a C-terminal-truncated protein that lacked part of the transmembrane domain and the two ATP-binding domains that causes a complete loss of $A B C C 6$ function. This substitution was not detected in the unaffected proband's wife and in 42 healthy Japanese volunteers in the JSNP database (http://snp.ims.u-tokyo.ac.jp/).

\section{Comments}

Although the family history was compatible with autosomal dominant inheritance, we also believe it is possible that the inheritance has a pseudo-dominant pattern (the vertical transmission of a recessive disorder occurring when the affected father marries a carrier).

Despite extensive screening, we have not found another mutation in the second, non-Q199X, ABCC6 allele in the siblings, leaving the possibility that mutations may be located in a non-coding region or consist of a heterozygous exonic deletion of the $A B C C 6$ gene.

A Medline search did not extract any report of the cooccurrence of RP and angioid streaks. We assume that the relationship between the two diseases observed in the siblings is coincidental, because of the significant difference in clinical expression, including fundus a

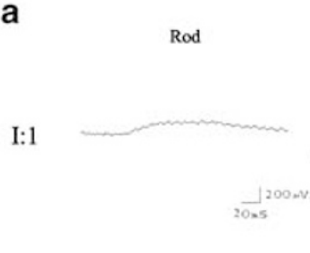

II:1

ПI:2
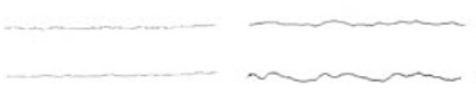

Normal

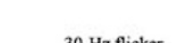

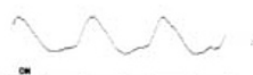

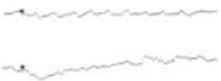

III: 12

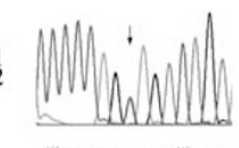

I:2

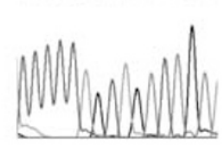

Figure 2 (a) Electrophysiological results in the affected patients and a normal subject. The scotopic rod ERG, 30-Hz flicker ERG, and bright-flash rod-cone mixed ERG amplitudes are markedly reduced in the siblings (II:1 and 2). Those of the proband are wellpreserved. The arrowheads indicate the stimulus onset. (b) Electropherogram of the sense strand of genomic DNA from the affected patients, showing a novel homozygous (I:1; top) or heterozygous (II:1 and 2; middle) nonsense mutation of $\mathrm{C}$ to $\mathrm{T}$ in exon 5 (Gln199stop). The arrows indicate the position of the mutation. The mutation was absent in the unaffected wife of the proband (I:2) (bottom). 
appearance and ERG findings, between the father and siblings. However, it is possible that different combinations of $A B C C 6$ alleles can give rise either to classic PXE or PXE and RP. It could also be possible that the mutation of the $A B C C 6$ in the siblings contributed to the pathogenesis of RP as a 'modifier gene' because $A B C A 4$ also encodes an ATP-binding cassette transporter, which has been responsible for RP. ${ }^{5}$ Although further study is needed to determine the genetic background of the PXE and RP observed in the siblings, our observations demonstrated the association of a novel ABCC6 mutation in Japanese patients with PXE, and point to the usefulness of web-based information of single-nucleotide polymorphism in extracting disease-causing mutations.

\section{Acknowledgements}

We thank Dr Franziska Ringpfeil (Jefferson Medical College, Philadelphia, PA, USA) for providing primer sequence information of $A B C C 6$.

\section{References}

1 Hu X, Plomp AS, van Soest S, Wijnholds J, de Jong PT, Bergen AA. Pseudoxanthoma elasticum: a clinical, histopathological, and molecular update. Surv Ophthalmol 2003; 48: 424-438.

2 Ringpfeil F, Lebwohl MG, Christiano AM, Uitto J. Pseudoxanthoma elasticum: mutations in the MRP6 gene encoding a transmembrane ATP-binding cassette (ABC) transporter. Proc Natl Acad Sci USA 2000; 97: 6001-6006.

3 Le Saux O, Urban Z, Tschuch C, Csiszar K, Baechelli B, Quaglino D. Mutations in a gene encoding an ABC transporter cause pseudoxanthoma elasticum. Nat Genet 2000; 25: 223-227.

4 Yoshida S, Kumano Y, Yoshida A, Numa S, Yabe N, Hisatomi T. Two brothers with gelatinous drop-like dystrophy at different stages of the disease: role of mutational analysis. Am J Ophthalmol 2002; 133: 830-832.

5 Martinez-Mir A, Paloma E, Allikmets R, Ayuso C, del Rio T, Dean M. Retinitis pigmentosa caused by a homozygous mutation in the Stargardt disease gene ABCR. Nat Genet 1998; 18: $11-12$. 